\title{
Variants in Scavenger Receptor Class B Type I Gene Are Associated with HDL Cholesterol Levels in Younger Women
}

\author{
Caroline G.P. Roberts $^{\mathrm{a}}$ Haiqing Shen ${ }^{\mathrm{b}}$ Braxton D. Mitchell ${ }^{\mathrm{b}}$ \\ Coleen M. Damcott ${ }^{b}$ Alan R. Shuldiner ${ }^{b, c}$ Annabelle Rodriguez ${ }^{a}$ \\ ${ }^{a}$ Department of Medicine, The Johns Hopkins University School of Medicine; ${ }^{b}$ Department of Medicine, \\ The University of Maryland School of Medicine, and ' Department of Veterans Affairs and Veterans Affairs \\ Medical Center Baltimore Geriatric Research, Education and Clinical Center (GRECC), Baltimore, Md., USA
}

\section{Key Words}

Atherosclerosis · Autosomal SNPs · SNP

\begin{abstract}
Objective: Variants within the scavenger receptor class B type I (SCARB1) receptor gene have been previously associated with lipid levels, especially in women, with some studies reporting the association to be stronger in the presence of diabetes or post-menopausal estrogen use. Based on the reported gender-specific association and modification effect of estrogen on lipid levels according to SCARB1 variants, we explored the relationship between SCARBI single nucleotide polymorphisms (SNPs) and lipid levels in an Amish population to assess sex and age differences. Methods: Eight SCARB1 SNPs, identified from public databases, were genotyped in 919 subjects. Results: Rs5888 and rs3782287 were in high linkage disequilibrium (LD), with $r^{2}>0.8$. None of the SNPs were significantly associated with lipid levels in men; however in women, rs5888 ( $p=0.04)$ and rs5891 $(p<0.001)$ were significantly associated with higher HDL-C levels. Rs5891 had an allele frequency of 3\% and predicts a mis-
\end{abstract}

C.G.P. Roberts and H. Shen are co-first authors.

\section{KARGER}

Fax +41613061234 E-Mail karger@karger.ch www.karger.com
(C) 2007 S. Karger AG, Base

0001-5652/07/0642-0107\$23.50/0

Accessible online at:

www.karger.com/hhe sense mutation (lle135Val), which may be functional. Moreover, rs3782287 ( $p=0.023)$ and rs5888 $(p=0.003)$ were significantly associated with higher HDL-C levels in women younger than 50 years but not in women aged 50 years or older ( $p$ for interaction between age and rs5888 $=0.045$ ). None of the SNP effects on HDL-C were modified in the presence of diabetes, in either men or women. Conclusions: SCARB1 SNPs influence HDL-C levels in women, particularly in those less than 50 years old. Condensed Abstract: We assessed associations between SCARB1 SNPs and lipid traits in 919 Amish men and women. Two SNPs, rs3782287 and rs5888, were significantly associated with higher HDL-C levels in women younger than 50 years but not in women aged 50 years or older, supporting an interaction between common sequence variants in SCARB1 and estrogen on HDL-C.

Copyright $\odot 2007$ S. Karger AG, Basel

The metabolism of high-density lipoprotein (HDL) is complex, with many factors influencing its circulating plasma levels. One such factor is the scavenger receptor, class B, type I (SR-BI), first characterized by Acton et al. [1], and subsequently shown to be a physiologically relevant receptor that exerts a major influence on HDL cho-

Dr. Annabelle Rodriguez

The Johns Hopkins Bayview Medical Center, Division of Endocrinology

Mason F. Lord Building, Center Tower, Room 639, 5200 Eastern Avenue

Baltimore, MD 21224 (USA)

Tel. +1 410550 8487, Fax +1 410550 3219, E-Mail arodrig5@jhmi.edu 
lesterol (HDL-C) levels in rodents [2]. It is highly expressed in the liver and in steroidogenic tissues $[1,3]$, where its primary function is to mediate the uptake of cholesteryl esters from the HDL particle core.

Much less is known regarding the role of SR-BI in humans, although recent studies have shown some variants in the $S R-B I$ gene (SCARB1) to be associated with lipid levels, lipoprotein particle size and body mass index (BMI) [4-8]. SCARB1 variants have also been studied in populations characterized with type 2 diabetes mellitus (T2DM) [5, 9] and, interestingly, a SCARB1 variant in exon 1 was found to associate with differences in insulin sensitivity in healthy people during the consumption of an olive oil-rich diet [10]. In addition, a SCARB1 variant was studied in older women using hormone replacement therapy [11]. Osgood et al. [9] examined SCARB1 gene variants in the participants of the Framingham Study, and reported that three common single nucleotide polymorphisms (SNPs) rs4238001 $($ exon $1,+4 \mathrm{G} \rightarrow \mathrm{A}$ ), rs5888 (exon $8,+63519 \mathrm{C} \rightarrow \mathrm{T}$ ) and an intron $5(+51905 \mathrm{C} \rightarrow \mathrm{T}) \mathrm{SNP}$ were associated with variation in plasma lipoprotein concentrations and particle size, particularly in subjects with T2DM. While there was no association of SNPs with T2DM, the G allele in rs4238001 was significantly associated with lower HDL-C levels and smaller HDL particle sizes, although only among subjects with T2DM. No interaction was observed for the rs5888 SNP with T2DM, whereas the interaction between the intron $5 \mathrm{SNP}$ and T2DM on HDL metabolism was seen only in men.

Gender-specific associations have been reported between some of the SCARB1 variants and lipid levels, an observation that led Richard et al. [11] to examine the effect of exogenous estrogen on HDL-Clevels bySCARB1 variants in elderly women in the Rancho Bernardo Study. These investigators found no significant associations between either the intron 5 SNP or rs5888 and HDL-C levels after adjusting for age, BMI, alcohol, smoking, triglycerides, fasting plasma glucose and estrogen use. However, they did report significant estrogen interaction with the rs5888 SNP on HDL-C levels, with HDL-C levels significantly higher in estrogen users than non-users [11].

Motivated by these previous observations, we assessed the relationship of eight different SCARB1 variants, including the previously studied rs 4238001 , intron $5(+51905$ $\mathrm{C} \rightarrow \mathrm{T}$ ), and rs5888 variants, with lipid levels in adult men and women participants of the Amish Family Diabetes Study (AFDS) and to determine if associations were modified by the effects of age, gender and/or T2DM.

\section{Methods}

\section{Study Population}

The Old Order Amish (OOA) is a genetically homogenous population of Central European ancestry. The original founders of this population immigrated to eastern Pennsylvania (now Lancaster County, Pa., USA) in the mid to late 1700 s, with the Lancaster County population now consisting of approximately 30,000 individuals [12]. The OOA today are still characterized by their social cohesiveness and rural lifestyle [13]. In 1995, AFDS was begun to identify susceptibility genes for T2DM and related traits. Probands with T2DM onset between 35 and 65 years of age were identified and all willing first-degree family members 18 years of age and older were invited to participate. First-degree relatives of any family members subsequently diagnosed with T2DM were also invited to participate. A total of 919 subjects with lipid data and DNA available for genotyping were included in this study.

Participating subjects were examined at the Amish Research Clinic in Strasburg, PA following a 12-hour overnight fast. Height and weight were measured and body mass index (BMI) was calculated as the weight in kilograms divided by the height in meters squared $\left(\mathrm{kg} / \mathrm{m}^{2}\right)$. Fasting lipid profiles [total cholesterol (TC), HDL-C, triglycerides (TG)] were performed by Quest Diagnostics (Baltimore, MD). Intra-assays CVs of duplicate samples ranged between 0.1 and $3.0 \%$, and inter-assay CVs ranged between 0.2 and 5\%. Low-density lipoprotein cholesterol (LDL-C) concentrations were estimated using Friedewald's formula [14].

A $75 \mathrm{~g}$ oral glucose tolerance test (OGTT) was administered, and diabetes mellitus was classified based on the American Diabetes Association plasma glucose criteria [15] of a fasting plasma glucose level ( $\geq 7 \mathrm{mmol} / \mathrm{l}$ ) or a 2-hour OGTT plasma glucose level ( $\geq 11.1 \mathrm{mmol} / \mathrm{l})$. Subjects were also considered to have diabetes if they reported current use of insulin or oral glucose-lowering agents. Subjects were excluded from these analyses if they had a diagnosis of diabetes before the age of 35 years and reported current use of insulin $(n=1)$. Impaired glucose tolerance was diagnosed based on OGTT plasma glucose levels (2-hour OGTT plasma glucose level $\geq 7.8$ but $<11.1 \mathrm{mmol} / \mathrm{l})$. Normal glucose tolerance was defined as fasting plasma glucose level $(<6.1 \mathrm{mmol} / \mathrm{l})$ and 2-hour OGTT plasma glucose level $(<7.8 \mathrm{mmol} / \mathrm{l})$.

Informed consent was obtained from all AFDS participants, and the study protocol was approved by the Institutional Review Board at the University of Maryland School of Medicine. Details of the study design have been previously described [13].

\section{Genotyping}

The SCARB1 gene is $93.2 \mathrm{~kb}$ in length and consists of 13 exons. We selected SNPs for genotyping within the coding and intronic regions of SCARB1 from public databases (NIH dbSNP, Wellcome Trust Sanger Institute and Applied Biosystems) that were validated by frequency (minor allele frequency $\geq 1 \%$ ) as well as SNPs reported in published articles $[4-8,16]$. SNPs were genotyped using the SNPstream UHT Genotyping System (Beckman Coulter, Fullerton, CA) [17]. Eight of the eighteen SNPs genotyped were polymorphic in this sample: rs3782287, rs5888, rs5890, rs5891, rs838884, rs10846760, rs10846745, rs11057851 and the intron 5 $(+51905 \mathrm{C} \rightarrow \mathrm{T})$ SNP. Error rates based upon blind replicates were $0-4.7 \%$. 
Table 1. Characteristics (mean $\pm \mathrm{SD}, \%$ ) of the Amish Family Diabetes Study cohort

\begin{tabular}{lccc}
\hline & Men & Women & Age-adjusted p \\
\hline $\mathrm{n}$ & 428 & 491 & \\
Age & $46.8 \pm 15.8$ & $47.1 \pm 16.2$ & 0.29 \\
$\mathrm{BMI}, \mathrm{kg} / \mathrm{m}^{2}$ & $26.3 \pm 4.0$ & $27.9 \pm 5.6$ & $<0.0001$ \\
Diabetes & $9.8 \%$ & $14.2 \%$ & 0.043 \\
Impaired glucose tolerance & $10.5 \%$ & $19.4 \%$ & 0.0002 \\
Total cholesterol, mg/dl & $213.2 \pm 48.6$ & $214.2 \pm 48.9$ & 0.86 \\
LDL-C, mg/dl & $149.6 \pm 44.0$ & $143.0 \pm 43.7$ & 0.013 \\
HDL-C, mg/dl & $46.9 \pm 12.7$ & $53.9 \pm 13.0$ & $<0.0001$ \\
Triglycerides, mg/dl & $84.4 \pm 57.5$ & $86.5 \pm 59.2$ & 0.66 \\
Lipid-lowering medications, \% & $1.9 \%$ & $1.2 \%$ & 0.42 \\
Estrogen users, \% & $\mathrm{NA}$ & $0.4 \%$ & $\mathrm{NA}$ \\
Oral hypoglycemic agents, \% & $1.9 \%$ & $3.0 \%$ & 0.25 \\
Insulin therapy, \% & $0.9 \%$ & $1.0 \%$ & 0.90 \\
\hline
\end{tabular}

$\mathrm{NA}=$ not applicable.

\section{Statistical Analysis}

Genotypes were checked for Mendelian consistency using the PedCheck software program in the extended Amish pedigree [18]. A small number of Mendelian errors were resolved or removed prior to analysis. The distribution of all SNP genotypes conformed to those expected under Hardy-Weinberg equilibrium using the $\chi^{2}$ test.

Association analyses were performed using a variance components methodology implemented in the Sequential Oligogenic Linkage Analysis Routines (SOLAR) software program, which allowed us to account for the relatedness of study subjects [19]. Briefly, we evaluated the effects of SNP genotype on HDL levels, while simultaneously adjusting for the effects of age, age ${ }^{2}$, sex, BMI, and diabetes status. We accounted for the non-independence among family members by modeling the residual correlations in HDL levels between relative pairs explicitly as a function of the kinship matrix. Our primary analysis was based on an additive genetic model, which implies a dose-response relationship between number of 'risk' alleles and HDL level. Genotypes were thus assigned values of 0,1 , or 2 , corresponding to the number of risk alleles. Subjects currently taking lipid-lowering medications $(\mathrm{n}=14)$ were excluded from the analysis. The significance of the genotype effects was assessed using the likelihood ratio test, in which we compared the likelihood of the data under a model in which the genotype effect was estimated against the likelihood of a nested model in which the genotype effect was constrained to zero.

Based upon our a priori hypothesis regarding an interaction between SCARB1 genotype and age or sex, genotype-phenotype associations were estimated in men and women (and older and younger women) separately. Interaction terms (e.g., genotype by sex, genotype by diabetes status, and genotype by age) were included in the model to assess whether the magnitude of the genotype effect on HDL levels differed by group.

Given negligible LD between SNPs (except rs5888 and rs3782287), haplotypes could not be reliably assigned, and thus haplotype association analysis was not performed.

\section{Results}

The 919 study subjects included 103 T2DM and 816 non-T2DM individuals from 226 families, ranging in size from 1 to 169. The characteristics of the study cohort are summarized in table 1 . Overall, there were slightly more women than men (53 vs. $47 \%$ ). Women had higher BMI (27.9 vs. $26.3 \mathrm{~kg} / \mathrm{m}^{2}, \mathrm{p}<0.0001$ ), higher prevalence of T2DM (14.2 vs. $9.8 \%, \mathrm{p}<0.043$ ), higher mean HDL-C levels (53.9 vs. $46.9 \mathrm{mg} / \mathrm{dl}, \mathrm{p}<0.0001)$ and lower mean LDL-C levels ( 143.0 vs. $149.6 \mathrm{mg} / \mathrm{dl}, \mathrm{p}<0.013$ ). The use of prescription medications such as lipid-lowering agents and hormone replacement therapy is low in the Amish. The frequency of cigarette smoking is also low in the Amish. Physical activity levels in the Old Order Amish are typically high, especially compared to non-Amish Caucasians. The Amish diet is generally high in calories and fat.

We next examined the associations between SCARB1 SNPs and lipid levels stratified by sex. In these analyses, we adjusted for age, age ${ }^{2}$, BMI, diabetes status, family structure and excluded those using lipid-lowering medications. None of the SCARB1 SNPs were associated with TC, HDL$\mathrm{C}$, LDL-C, or TG levels in men (data not shown). However, in women, rs5888 $(\mathrm{p}=0.04)$ and $\mathrm{rs} 5891(\mathrm{p}<0.001)$ were significantly associated with higher HDL-C levels (fig. 1). The genotype $\times$ gender interaction was significant for rs5888 using a dominant model ( $\mathrm{p}<0.005)$, whereas no significant interaction was found for rs5891. As the Rancho Bernardo study [11] suggested an interaction between estrogen use and rs5888 SNP genotypes on HDL-C and because estrogen levels are related to age, we examined as- 
Fig. 1. Gene structure and pairwise LD among SNPs in SCARB1. The upper portion of the figure shows the gene structure and location of polymorphisms. The lower portion of the figure shows a schematic pairwise $\mathrm{LD}$, calculated as $\mathrm{D}^{\prime}$ and $\mathrm{r}^{2}$ among the polymorphic SNPs in the Amish. The lines connect each SNP name and the position with the corresponding cell in the LD matrix. Magnitude and significance of the $\mathrm{D}^{\prime}$ and $\mathrm{r}^{2}$ is illustrated by shading with a red to white or a blue to white gradient reflecting higher to lower LD values.

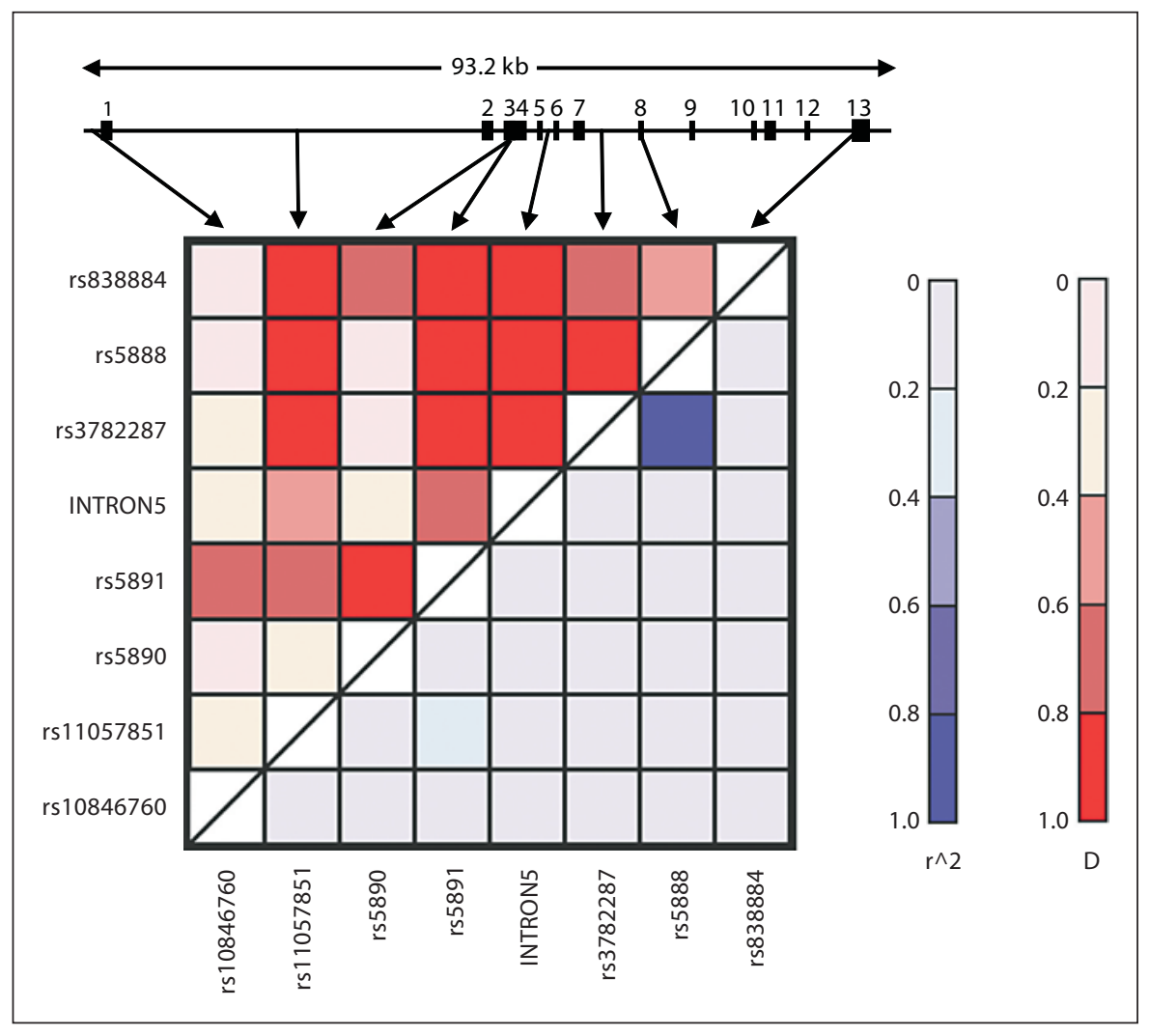

Table 2. Mean HDL-C levels (SE) according to SCARB1 SNP genotype in women $\geq 50$ and $<50$ years of age

\begin{tabular}{|c|c|c|c|c|c|c|c|c|c|}
\hline \multirow[t]{2}{*}{ SNP name } & \multirow[t]{2}{*}{ MAF } & \multicolumn{4}{|c|}{ Women $\geq 50$ years $(n=192)$ : Genotype } & \multicolumn{4}{|c|}{ Women $<50$ years $(\mathrm{n}=293)$ : Genotype } \\
\hline & & 11 & 12 & 22 & $\mathrm{p}^{*}$ & 11 & 12 & 22 & $\mathrm{p}^{*}$ \\
\hline rs10846760 (C/T) & 0.25 & $54.4(6.4)$ & $55.6(6.4)$ & $60.9(6.4)$ & 0.34 & $53.6(4.4)$ & $53.7(4.4)$ & $62.4(4.4)$ & 0.33 \\
\hline rs11057851 (G/A) & 0.08 & $55.1(1.5)$ & $59.4(2.7)$ & $45.2(12.3)$ & 0.25 & $53.5(1.2)$ & $53.3(2.2)$ & & 0.92 \\
\hline rs5890 $(\mathrm{C} / \mathrm{T})$ & 0.003 & $54.9(12.5)$ & $63.4(12.4)$ & & 0.50 & $53.5(7.0)$ & $44.1(7.1)$ & & 0.19 \\
\hline rs5891 (G/A) & 0.03 & $54.6(1.4)$ & $65.5(4.2)$ & $64.8(12.1)$ & 0.01 & $53.0(1.2)$ & $61.1(3.1)$ & & 0.01 \\
\hline Intron5 $(\mathrm{T} / \mathrm{C})$ & 0.05 & $55.5(1.4)$ & $54.2(2.9)$ & & 0.66 & $54.4(1.2)$ & $54.1(2.7)$ & & 0.89 \\
\hline rs3782287 (A/G) & 0.41 & $54.3(2.8)$ & $57.1(2.6)$ & $52.5(2.6)$ & 0.84 & $50.2(2.4)$ & $54.2(2.2)$ & $54.9(2.1)$ & 0.02 \\
\hline rs5888 $(\mathrm{C} / \mathrm{T})$ & 0.41 & $53.3(2.7)$ & $56.9(2.5)$ & $52.5(2.5)$ & 0.89 & $49.6(2.4)$ & $54.4(2.1)$ & $56.1(2.0)$ & 0.003 \\
\hline rs838884 (T/C) & 0.23 & $56.2(1.6)$ & $54.7(1.9)$ & $55.0(4.3)$ & 0.86 & $54.7(1.3)$ & $55.8(1.6)$ & $45.8(3.7)$ & 0.72 \\
\hline
\end{tabular}

${ }^{*} \mathrm{p}$ is for additive genetic model, adjusted for age, BMI, family structure, diabetes, and excluded lipid medications. Significant $\mathrm{p}$ values are printed in bold.

sociations between SCARB1 SNPs and HDL-C levels in women older and younger than age 50 years. As shown in table 2, women less than 50 years of age who carried the rs5888 ( $\mathrm{p}<0.003)$ or rs3782287 ( $<<0.023)$ minor allele had significantly higher HDL-C levels compared with women homozygous for the major allele (HDL-C levels ap- proximately 13 and $9 \%$ higher, respectively). In women greater than 50 years of age, these two SNPs were not associated with HDL-C levels. The genotype $\times$ age group interaction term was statistically significant for rs5888 genotypes $(p=0.045)$. By contrast, rs5891, which encodes a missense mutation (Ile135Val), was associated with HDL- 
C levels in women from both age groups, although this SNP was uncommon (3\%) in this population. None of the SNPs were associated with diabetes, in either men or women, nor were the effects of any SNP on HDL-C levels modified in the presence of diabetes. Excluding subjects with diabetes did not appreciably change the HDL-C association results (data not shown).

A description of the gene structure showing SNP locations and pairwise LD between SNPs is provided as figure 2. Rs5888 (exon 8; silent) and rs3782287 (intron 7), both of which were associated with HDL-C levels in women younger than 50 years old, are located $4.5 \mathrm{~kb}$ from each other and are in high LD with each other. By contrast, rs5891 (exon 3; Ile135Val), which was also associated with HDL-C levels, is rarer and not linked to the other two HDL-C-associated SNPs, suggesting that its association with HDL-C levels is independent of the other two SNPs. This was supported by the fact that the association of rs5891 with HDL-C levels remained statistically significant even after adjusting for the effects of the other two SNPs in either total women or women of each age group. The opposite analyses were done on rs5888 or rs 3782287 while adjusting for rs5891. Significant association between rs5888 or rs3782287 and HDL-C remained in total women and women younger than 50 years old (data not shown). Because of the relative absence of LD between all pairs of SNPs $\left(\mathrm{r}^{2} \leq 0.20\right)$, with the exception of SNPs rs5888 and rs3782287, which were in very high $\mathrm{LD}\left(\mathrm{r}^{2}=0.86\right)$, no haplotype association analyses were performed.

\section{Discussion}

We have observed several SCARB1 polymorphisms to be associated with HDL-C levels in Amish women, but not in men. Furthermore, the associations were stronger in younger as compared to older women. Because Amish women, at least in Lancaster County, rarely take estrogen ( $0.41 \%$ in our cohort), the stratification of women according to those younger and older than age 50 years is a proxy for examining the interaction of estrogen on lipid levels according to SCARB1 genotype. Our results are consistent with Richard et al. [11] in that HDL-C levels were significantly higher in Amish women younger than 50 years of age (presumably pre-menopausal and having adequate endogenous estrogen levels) who carried the rs5888 variant, suggesting an important interaction between estrogen and SR-BI. In addition, we found significantly higher HDL-C in younger female carriers of the minor allele of rs3782287. However, unlike the Rancho

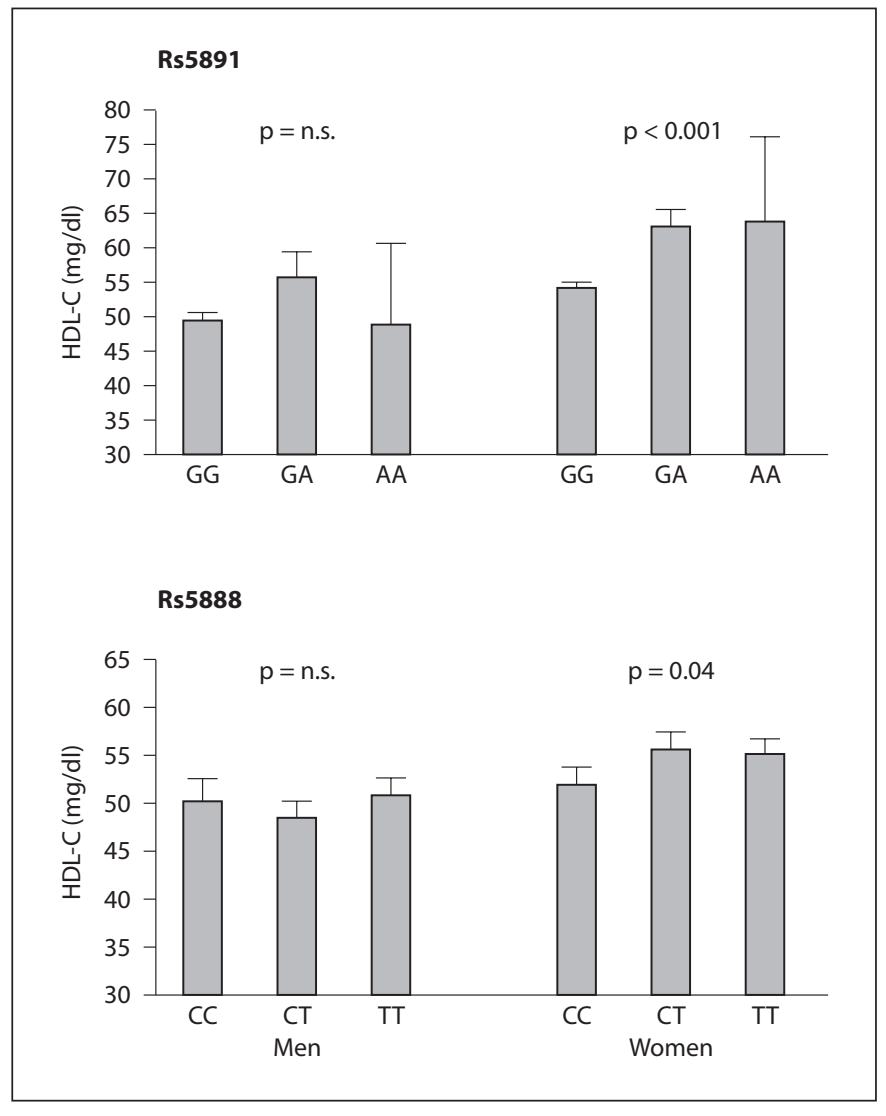

Fig. 2. Adjusted HDL-C levels in men and women according to rs5888 and rs5891 genotype. Mean HDL-C levels for men and women according to rs5888 and rs5891 genotype are shown. The $p$ value for women is statistically significant for trend while the $p$ value for men is not significant. There is a significant gene $\times$ gender interaction for rs5888 ( $\mathrm{p}<0.005)$.

Bernardo population, we did not find a difference in LDL-C levels in the Old Order Amish women according to age and SCARB1 genotype.

Osgood et al. [9] did not show a sex difference between lipid levels and the rs5888 SNP. After multivariate adjustments, they showed that men homozygous for the minor allele had significantly higher total HDL-C, higher $\mathrm{HDL}_{3}-\mathrm{C}$ levels and larger HDL particle size compared with men homozygous for the major allele. However, in women, while there were no apparent differences in HDL-C levels between minor allele homozygotes and major allele homozygotes, LDL-C levels were lower and HDL particle size was larger in the minor allele homozygotes [9]. Acton et al. [4] also did not find significant as- 
sociations between lipid levels and the rs5888 SNP in men in a population sampled from Zaragoza, Spain. While they also did not find significant associations between HDL-C levels and rs5888 variant in women, they reported that women carrying the minor allele had significantly lower LDL-C levels compared to women homozygous for the major allele. Other groups examining the rs5888 SNP have also reported association of this SNP with lipid levels $[5,6]$. Thus, our findings, coupled with reports in the literature, provide evidence for replicated association of rs5888 with lipid levels, although there appear to be inconsistencies with respect to which lipid traits.

In our population we did not find associations between SCARB1 SNPs and triglycerides (p ranges 0.08 0.8 ). Only one SCARB1 SNP was significantly associated

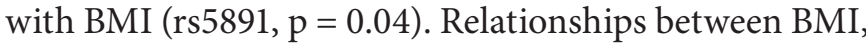
triglycerides, and SCARB1 SNPs have been found in a Spanish population [4], and relationships between triglycerides and the SCARB1 intron 5 SNP have been described in an obese population undergoing gastric bypass surgery [20]. These differences may be due to the different populations studied.

The relationship between SCARB1 and estrogen may have biological relevance given that in animals estrogen regulates the expression of SR-BI and its isoform SR-BII $[21,22]$, and that estrogen response elements are found within the SCARB1 promoter [23, 24]. In humans, we have shown that women undergoing oocyte retrieval for in vitro fertilization who have low estradiol levels are also low expressors of SR-BI mRNA [25]. It is possible that rs5888 or rs3782287 may be in linkage disequilibrium with a functional variant in estrogen response element in $S C A R B 1$ such that carriers of variant alleles for SCARB1 may have altered expression of SR-BI that becomes apparent only in the setting of estrogen. Interestingly, rs5891, which encodes a missense mutation (Ile135Val), is associated with HDL-C levels in both age groups. Although rare, it is possible that the effect of this potentially functional variant is strong enough to be independent of estrogen status.

The limitations of our study merit further discussion. The OOA are a closed founder population with relatively homogeneous environmental factors and fewer confounders of disease and related phenotypes (e.g., low rates of smoking, alcohol consumption, and medication usage) [13]. Although this homogenous lifestyle might enhance our ability to discern genetic influences on disease and related traits, the ability to generalize our findings will require further study in the general population. In addition, the high fat diet of the OOA (approximately $36 \%$ saturated fat (Shuldiner, in preparation)) may also contribute to an interaction between diet, genotype and lipid levels. Perez-Martinez explored the effect of dietary fat content (saturated fat diet, carbohydrate-rich diet and a monounsatured olive oil diet) on lipid levels in subjects stratified by the SCARB1 exon 1 SNP genotype. Subjects consuming a saturated fat diet and expressing the minor allele in exon 1 had significantly higher LDL-C levels compared to subjects homozygous for the major allele [26]. This suggests the possibility that dietary fat might also have an important interaction with other SCARB1 variants. Furthermore, Spady et al. [27] have previously shown that polyunsaturated fatty acids up-regulate SR-BI in the hamster.

It should be noted that we reported nominal $\mathrm{p}$ values in our statistical analysis instead of $\mathrm{p}$ values adjusted for multiple comparisons. However, since a major motivation of our study was to determine if observed associations between SCARB1 SNPs and HDL-C levels were influenced by age and gender given prior speculations that genetic effects at this locus might be estrogen sensitive, the comparisons we considered were not wholly independent. Nonetheless, we could not exclude the possibility of a false-positive association and we need to be cautious in our interpretation of the results.

In summary, we have shown that some SCARB1 variants are significantly associated with HDL-C levels in participants of the AFDS. One of these variants encodes a relatively rare conservative missense mutation and thus may be functional. Interestingly, there was a significant age or estrogen interaction with two more common noncoding SCARB1 variants. The clinical relevance of these associations and the mechanism by which these variants influence HDL-C levels, especially in younger women, warrants further study.

\section{Acknowledgments}

This work was supported by research grants R01-DK54261, U01-DK58026, and K07-CA67960 awarded to the University of Maryland and to HL075646 awarded to Dr. Annabelle Rodriguez. Other support was received from the University of Maryland General Clinical Research Center Grant M01 RR 16500; the General Clinical Research Centers Program; the National Center for Research Resources (NCRR); the National Institutes of Health; The NIDDK Clinical Nutrition Research Unit of Maryland (NIH P30 DK072488); and the Baltimore Veterans Administration Geriatric Research and Education Clinical Center. We gratefully acknowledge our Amish liaisons and fieldworkers and the extraordinary cooperation and support of the Amish community, without whom these studies would not be possible.
Roberts/Shen/Mitchell/Damcott/ Shuldiner/Rodriguez 


\section{References}

- 1 Acton S, Rigotti A, Landschulz KT, Xu S, Hobbs $\mathrm{HH}$, Krieger M: Identification of scavenger receptor SR-BI as a high density lipoprotein receptor. Science 1996;271:518520.

-2 Rigotti A, Trigatti BL, Penman M, Rayburn H, Herz J, Krieger M: A targeted mutation in the murine gene encoding the high density lipoprotein (HDL) receptor scavenger receptor class B type I reveals its key role in HDL metabolism. Proc Natl Acad Sci USA 1997; 94:12610-12615.

- 3 Trigatti B, Rayburn H, Vinals M, Braun A, Miettinen H, Penman M, Hertz M, Schrenzel M, Amigo L, Rigotti A, Krieger M: Influence of the high density lipoprotein receptor SR-BI on reproductive and cardiovascular pathophysiology. Proc Natl Acad Sci USA 1999;96:9322-9327.

-4 Acton S, Osgood D, Donoghue M, Corella D, Pocovi M, Cenarro A, Mozas P, Keilty J, Squazzo S, Woolf EA, Ordovas JM: Association of polymorphisms at the SR-BI gene locus with plasma lipid levels and body mass index in a white population. Arterioscler Thromb Vasc Biol 1999;19:1734-1743.

5 McCarthy JJ, Lewitzky S, Reeves C, Permutt A, Glaser B, Groop LC, Lehner T, Meyer JM: Polymorphisms of the HDL receptor gene associated with HDL cholesterol levels in diabetic kindred from three populations. Hum Hered 2003;55:163-170.

-6 McCarthy JJ, Lehner T, Reeves C, Moliterno DJ, Newby LK, Rogers WJ, Topol EJ; Genequest investigators: Association of genetic variants in the HDL receptor, SR-B1, with abnormal lipids in women with coronary artery disease. J Med Genet 2003;40:453-458.

7 Hsu LA, Ko YL, Wu S, Teng MS, Peng TY, Chen CF, Chen CF, Lee YS: Association between a novel 11-base pair deletion mutation in the promoter region of the scavenger receptor class B type I gene and plasma HDL cholesterol levels in Taiwanese Chinese. Arterioscler Thromb Vasc Biol 2003;23:18691874.

8 Rodriguez-Esparragon F, Rodriguez-Perez JC, Hernandez-Trujillo Y, Macias-Reyes A, Medina A, Caballero A, Ferrario CM: Allelic variants of the human scavenger receptor class $B$ type 1 and paraoxonase 1 on coronary heart disease: genotype-phenotype correlations. Arterioscler Thromb Vasc Biol 2005; $25: 854-860$. $\checkmark 9$ Osgood D, Corella D, Demissie S, Cupples LA, Wilson PW, Meigs JB, Schaefer EJ, Coltell O, Ordovas JM: Genetic variation at the scavenger receptor class B type I gene locus determines plasma lipoprotein concentrations and particle size and interacts with type 2 diabetes: The Framingham Study. J Clin Endocrinol Metab 2003;88:2869-2879.

10 Perez-Martinez P, Perez-Jimenez F, Bellido C, Ordovas JM, Moreno JA, Marin C, Gomez P, Delgado-Lista J, Fuentes F, Lopez-Miranda J: A polymorphism exon 1 variant at the locus of the scavengar receptor class B type I (SCARB1) gene is associatred with differences in insulin sensitivity in healthy people during the consumption of an olive oil-rich diet. J Clin Endocrinol Metab 2005;90:22972300.

11 Richard E, von Muhlen D, Barrett-Connor E, Alcaraz J, Davis R, McCarthy JJ: Modification of the effects of estrogen therapy on HDL cholesterol levels by polymorphisms of the HDL-C receptor, SR-BI: the Rancho Bernardo Study. Atherosclerosis 2005;180:255262.

12 Beiler K: Church Directory of the Lancaster County Amish. Gordonsville, PA, Peqaea Publishers.

13 Hsueh WC, Mitchell BD, Aburomia R, Pollin T, Sakul H, Gelder Ehm M, Michelsen BK, Wagner MJ, St Jean PL, Knowler WC, Burns DK, Bell CJ, Shuldiner AR: Diabetes in the Old Order Amish: Characterization and heritability analysis of the Amish Family Diabetes Study. Diabetes Care 2000;23:595-601.

14 Friedewald WT, Levy RI, Fredrickson DS: Estimation of the concentration of low-density lipoprotein cholesterol in plasma, without use of the preparative ultracentrifuge. Clin Chem 1972;18:499-502.

15 Expert Committee on the Diagnosis and Classification of Diabetes Mellitus: Followup report on the diagnosis of diabetes mellitus. Diabetes Care 2003;26:3160-3166.

-16 Osgood-McWeeney D, Galluzzi JR, Ordovas JM: Allelic discrimination for single nucleotide polymorphisms in the human scavenger receptor class $B$ type 1 gene locus using fluorescent probes. Clin Chem 2000;46:118119.

17 Bell PA, Chaturvedi S, Gelfand CA, Huang CY, Kochersperger M, Kopla R, Modica F, Pohl M, Varde S, Zhao R, Zhao X, BoyceJacino MT, Yassen A: SNPstream UHT: Ultra-high throughput SNP genotyping for pharmacogenomics and drug discovery. Biotechniques 2002;Suppl:70-72, 74, 76-77.
18 O'Connell JR, Weeks DE: PedCheck: A program for identification of genotype incompatibilities in linkage analysis. Am J Hum Genet 1998;63:259-266.

19 Almasy L, Blangero J: Multipoint quantitative-trait linkage analysis in general pedigrees. Am J Hum Genet 1998;62:11981211.

20 Koumanis DJ, Christou NV, Wang XL, Gilfix BM: Pilot study examining the frequency of several gene polymorphisms in a morbidly obese population. Obes Surg 2002;12:759764.

21 Landschulz KT, Pathak RK, Rigotti A, Krieger M, Hobbs $\mathrm{H}$ : Regulation of scavenger receptor, class B, type I, a high density lipoprotein receptor, in liver and steroidogenic tissues of the rat. J Clin Invest 1996;98:984995

22 Graf GA, Roswell KL, Smart EJ: 17 beta-estradiol promotes the up-regulation of SR-BII in HepG2 cells and in rat livers. J Lipid Res 2001;42:1444-1449.

23 Lopez D, McLean MP: Estrogen regulation of the scavenger receptor class B gene: antiatherogenic or steroidogenic, is there a priority? Mol Cell Endocrinol 2005; Nov 14, epub.

24 Lopez D, Sanchez MD, Shea-Eaton W, McLean MP: Estrogen activates the highdensity lipoprotein receptor gene via binding to estrogen response elements and interaction with sterol regulatory element binding protein-1A. Endocrinology 2002;143:21552168

25 Velasco M, Alexander C, King J, Zhao Y, Garcia J, Rodriguez A: Association of lower plasma estradiol levels and low expression of scavenger receptor class B, type I in infertile women. Fertil Steril 2006;85:1391-1397.

-26 Perez-Martinez P, Ordovas JM, Lopez-Miranda J, Gomez P, Marin C, Moreno J, Fuentes F, Fernandez de la Puebla RA, Perez-Jimenez F: Polymorphism exon 1 variant at the locus of the scavenger receptor class $\mathrm{B}$ type I gene: Influence on plasma LDL cholesterol in healthy subjects during the consumption of diets with different fat contents. Am J Clin Nutr 2003;77:809-813.

$>27$ Spady DK, Kearney DM, Hobbs HH: Polyunsaturated fatty acids up-regulate hepatic scavenger receptor B1 (SR-BI) expression and HDL cholesteryl ester uptake in the hamster. J Lipid Res 1999;40:1384-1394. 